\title{
Application of LPME/GC-ECD for determination of PCP in water
}

\section{Ping (李 萍) \& LIU Junxin (刘俊新)}

Research Center for Eco-Environmental Sciences, Chinese Academy of Sciences, Beijing 100085, China Correspondence should be addressed to Li Ping (email: liping15cn@yahoo.com.cn)

Received April 17, 2003

\begin{abstract}
Liquid-phase microextraction (LPME) followed by gas chromatography (GC) coupled with an electron capture detector (ECD) was applied for the analysis of pentachlorophenol (PCP) in aqueous samples. After alkalization with $\mathrm{Na}_{2} \mathrm{CO}_{3}$ solution, PCP was acetylated with acetic anhydride. The pentachlorophenyl acetate derivative was then extracted with $n$-hexane by LPME. 1.5 $\mu \mathrm{L}$ organic drop exposed to the aqueous sample solution at $15^{\circ} \mathrm{C}$ for $5 \mathrm{~min}$, stirring rate at 100 $\mathrm{r} / \mathrm{min}$ was chosen as the optimum extraction condition. Under this condition, LPME provided a very simple, fast and solvent-less procedure to collect PCP from aqueous sample for GC determination. The linearity of LPME/GC-ECD for PCP in distilled water was investigated at the range of 2-200 $\mu \mathrm{g} / \mathrm{L}$ with a coefficient of correlation 0.998 . The repeatability of this method was determined at the level of $50 \mu \mathrm{g} / \mathrm{L}$ with the average of relative standard deviations (RSDs) $7 \%$. Detection limit was obtained at less than $0.5 \mu \mathrm{g} / \mathrm{L}$ levels. When LPME/GC-ECD was applied to the determination of $\mathrm{PCP}$ in municipal sewage, the average of relative recoveries and RSDs were $70 \%$ and $6.5 \%$, respectively.
\end{abstract}

Keywords: liquid-phase microextraction, pentachlorophenol, municipal sewage.

DOI: 10.1360/03yb0030

PCP with a variety of application in industrial, agricultural and domestic fields, is an inhibitor of oxidative phosphorylation, therefore, toxic to a broad spectrum of organisms and may have mutagenic properties ${ }^{[1]}$. In China, sodium pentachlorophenate (Na-PCP) has been sprayed widely since the 1960s to control the spread of snailborne schistosomiasis. Although Na-PCP has been replaced by some new molluscicides since 1990s, the persistence of PCP has resulted in the accumulation in soil and sediments ${ }^{[2]}$.

To assess the possible impact of PCP on ecosystems and support the improvement of remediation techniques, a rapid, accurate and sensitive analytical method of identifying and determining trace PCP in sewage samples is highly desirable to be developed. Selecting the optimum methods of sample preparation is essential for trace analysis of PCP in sewage. The extraction efficiency from the complex matrix (particularly in sewage sample) affects the detection level.

LLE and SPE are the most commonly used techniques for preconcentration of aqueous sample for analysis. However, these methods are time consuming and tedious, particularly for LLE, which requires large volumes of aqueous samples and consumes a lot of organic solvents. SPE offers the advantages to consume much less solvent and to be automatisable, but SPE is relatively 
expensive. SPE requires substantial investment in equipment and extraction cartridges. These cartridges are not reusable and can plug if the samples contain solid particles. Solid-phase microextraction is a more recent procedure and has been developed for preconcentration various analytes from aqueous sample. It is fast and solvent-free. However, SPME also suffers from some problems such as sample carry-over, relatively high-cost and fiberfragility.

In LPME, which based on the use of small amount of organic solvent to extract analytes from moderate amount of aqueous matrices, the principles of LLE and the miniaturized nature of SPE are combined to realize the advantages of both techniques. This novel technique for sample extraction has been described in several papers, accomplished by extraction either into small water immiscible drops of organic solvents, called microdrop $\mathrm{LPME}^{[3-9]}$, or into small volumes of acceptor solution present inside the lumen of porous hollow fiber, also termed LPME/HF ${ }^{[10-12]}$.

For the LPME/HF, porous polypropylene hollow fiber with impregnated organic solvent was used as an interface between the donor and acceptor phase. The hollow fiber with walls of o.2 $\mu \mathrm{m}$ pore size provided a measure of sample clean-up because it prevented large molecules in donor phase from being extracted.

Comparing with LPME/HF, microdrop LPME provided the high preconcentration factors and was shown to be fast and highly efficient. However, this method is not suitable for "dirty" sample such as slurry and soil sample, because the particles in such solutions will damage the syringe.

In this paper, the optimum procedures of analyzing PCP in aqueous samples by microdrop LPME-GC-ECD and the feasibility of applying this technique to municipal sewage were systematically evaluated. The detection limits, linear dynamic ranges and reproducibility were also investigated.

\section{Materials and methods}

\subsection{Materials}

All chemicals used were of analytical grade or the best quality available. Distilled and deionized water was used throughout the experiment. Prior to use, all glassware was soaked in methanol solution overnight and then rinsed in tap water and distilled water and thoroughly dried.

PCP was dissolved in $0.1 \mathrm{~mol} / \mathrm{L}$ sodium hydroxide solvent to obtain a standard stock solution with the concentration of $600 \mathrm{mg} / \mathrm{L}$. They were stored at $4^{\circ} \mathrm{C}$ in a refrigeratory. The aqueous samples were prepared by spiking with stock standard PCP solution.

$100 \mathrm{~mL}$ of aqueous sample was transferred into a sample vial and $1 \mathrm{~g} \mathrm{Na}_{2} \mathrm{CO}_{3}$ was added. After alkalization, $0.5 \mathrm{~mL}$ of acetic anhydride was added. The sample was shaken for 2 min and let stand for $10 \mathrm{~min} .10 \mathrm{~mL}$ of aqueous sample was transferred into15 mL sample vial and used for LPME.

\subsection{Extraction procedure}

The LPME were performed in a device shown in fig. 1. A $15 \mathrm{~mL}$ sample vial containing 10 
$\mathrm{mL}$ sample solution equipped with a $0.8-\mathrm{cm}$ magnetic stir bar was placed in a water-jackted vessel and maintained at a constant temperature $\left(15^{\circ} \mathrm{C}\right)$ by a water bath. During an extraction, a 5 $\mu \mathrm{L}$ microsyringe was clamped above the sample vial so that the syringe needle tip was below the surface of the aqueous sample. The solution was stirred at $100 \mathrm{r} / \mathrm{min}$. The type of microsyringe employed here has a needle tip with an about $22^{\circ}$

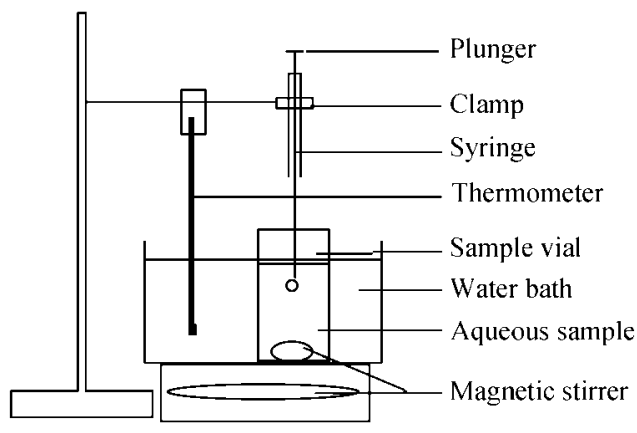

Fig. 1. Schematic of LPME device. bevel.

An aliquot ( $2 \mu \mathrm{L}$ ) of $n$-hexane was withdrawn into syringe. The plunge was depressed by 0.5 $\mu \mathrm{L}$ to produce a small drop at the tip of syringe, then, the needle tip was inserted through the sample vial septum and immersed into the $10 \mathrm{~mL}$ stirred sample solution to a depth of $1 \mathrm{~cm}$ below the surface. The syringe was held in place by clamp. The syringe plunge was depressed to expose a $1.5 \mu \mathrm{L}$ drop of solvent in aqueous sample. Extraction then occurred between this droplet and sample for $5 \mathrm{~min}$. The droplet was retracted into the syringe. After the syringe removed from the sample vial, the plunger was then depressed to the $1 \mu \mathrm{L}$ position and the needle tip was cleaned carefully with a tissue to remove possible water contamination. Finally, the extract was injected into the gas chromatograph.

\subsection{Detector}

Chromatographic analysis was performed on a 5890 chromatograph equipped with a split/ splittless injector, which was used in splittless mode and an electron-capture detection (ECD) system. Separations were conducted using an HP-5, $30 \mathrm{~m} \times 0.25 \mathrm{~mm}$ capillary column with a 0.25 $\mu \mathrm{m}$ stationary phase thickness. The carrier gas was purified nitrogen at a flowrate of $1.7 \mathrm{~mL} / \mathrm{min}$. The gas chromatography (GC) conditions were as follows: injector temperature $240^{\circ} \mathrm{C}$; detector temperature $260^{\circ} \mathrm{C}$; initial oven temperature $120^{\circ} \mathrm{C}$ for $5 \mathrm{~min}$, increased to $210^{\circ} \mathrm{C}$ at a rate of 30 ${ }^{\circ} \mathrm{C} / \mathrm{min}$, then maintained at $210^{\circ} \mathrm{C}$ for $12 \mathrm{~min}$. The total time for one GC run was $20 \mathrm{~min}$.

\section{Results and discussion}

\subsection{Optimization of LPME}

In this experiment, the organic drop is exposed to the aqueous sample solution. The analyte in aqueous solution is transferred into the organic drop by diffusion. In order to optimize the sample technique, several parameters affecting the sampling efficiency were studied by monitoring the variation of analytical signal with PCP concentrations. The optimization procedure of the experimental condition followed a described methodology ${ }^{[7]}$ that was developed for the analysis of organochlorine pesticides in water. In this paper, $1.5 \mu \mathrm{L}$ organic drop exposed to the aqueous sample solution at $15^{\circ} \mathrm{C}$ for $5 \mathrm{~min}$, and the stirring rate at $100 \mathrm{r} / \mathrm{min}$ was chosen as the optimum condition 
and used in the following experiment.

\subsection{Linearity and reproducibility}

In an analysis, it is desired to evaluate the initial concentration of analytes in an aqueous sample solution based upon the measured value of the analyte concentration in the organic drop at sampling time.

In this paper, five aqueous samples with different PCP concentrations were subjected to extraction by $1.5 \mu \mathrm{L}$ of $n$-hexane drops for $5 \mathrm{~min}$. $1 \mu \mathrm{L}$ of the extraction solution was measured by GC-ECD and the measured signals were plotted against the initial aqueous concentration of PCP. Therefore, when the concentrations of PCP in distilled water were 2, 10, 50, 100, $200 \mu \mathrm{g} / \mathrm{L}$, respectively, correspondingly the peak area values by the GC-ECD were 576.45, 2841.66, 13661.68, 23186.50, 46652.43. The calibration curves were obtained under the optimum experimental condition. Linearity was observed over the range of $2-200 \mu \mathrm{g} / \mathrm{L}$. Coefficient of correlation $\left(r^{2}\right)$ is 0.998 .

The reproducibility in peak responses was investigated on six replicate aqueous samples (50 $\mu \mathrm{g} / \mathrm{L}$ ) under the optimized condition. The average of relative standard deviations (RSDs) of PCP is 7\%. The average of RSDs is not completely satisfactory. There may be three possible reasons. First, under the experimental conditions, the partition equilibrium not reached, so precise timing is essential for good precision. Second, during the extraction procedure, the liquid organic drop dissolved trifling and the water of aqueous sample also diffused into organic solution slightly with increasing the exposure time. The stirring rate and extraction temperature also affect the precision. Third, if the organic drop could be drawn back into syringe complete after sampling, conceivably contributed to the reproducibility.

\subsection{Limit of detection}

Limitation of detections (LOD) is defined as the lowest concentrations of the analyte that produce chromatographic peaks. Based on a signal-to-noise ratio $\mathrm{S} / \mathrm{N}=3$, the LOD was evaluated under GC-ECD conditions. In this paper, the value of LOD was found to be in the range of 0.3$0.5 \mu \mathrm{g} / \mathrm{L}$, generally comparable to those US Environment Protection Agency (EPA) method 604 $(7.4 \mu \mathrm{g} / \mathrm{L})^{[13]}$. To determine the LOD, syringe blanks were carried out every time to confirm that no sample carryover occurred. By rinsing of the syringe with $n$-hexane again and again, the elimination of sample carryover is more effectively performed than SPME.

\subsection{Recovery}

Municipal sewage was spiked with PCP standard stock solution at the concentration range from 10 to $250 \mu \mathrm{g} / \mathrm{L}$ to assess matrix effects. Some of chromatograms are shown in fig.2.

The relative recovery is defined as the ratio of GC peak areas of spiked sewage sample extracts to spiked distilled water extracts. For municipal sewage, the average of relative recoveries is about $70 \%$ with the average of RSDs no more than $6.5 \%$, respectively. 

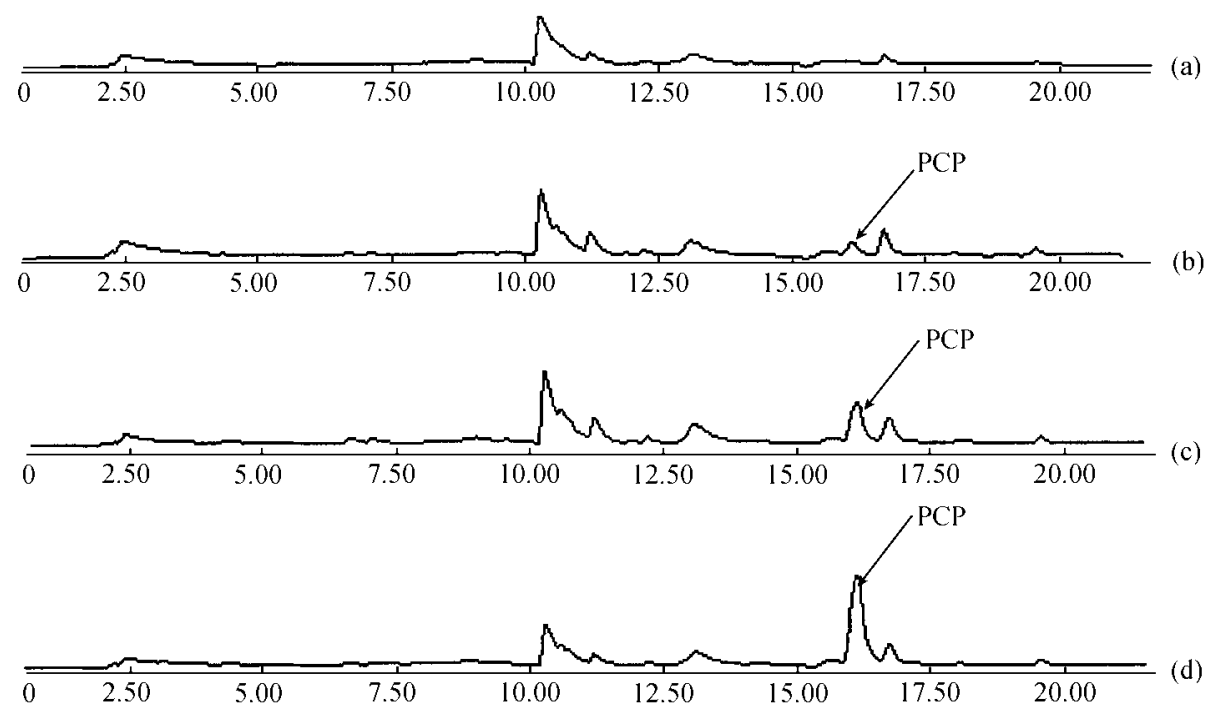

Fig. 2. Chromatograms of PCP in municipal sewage. (a) Spiked with $0 \mu \mathrm{g} / \mathrm{L}$, (b) spiked with $10 \mu \mathrm{g} / \mathrm{L}$, (c) spiked with 50 $\mu \mathrm{g} / \mathrm{L}$, (d) spiked with $100 \mu \mathrm{g} / \mathrm{L}$.

\section{Conclusions}

A microdrop of organic solvent is suspended directly at the tip of a microsyringe needle immersed in a stirred aqueous sample. After exposure for a prescribed time, the microdrop is retracted into the microsyringe and an aliquot of the solvent is injected into the gas chromatograph for analysis. The proposed solvent microextraction technique, LPME-GC-ECD, is attractive owing to its simplicity, analytical precision, short sample preparation time, low cost and minimization of organic solvent used. The experiment results show that this method has been illustrated to be viable, easy to use, and rapid for qualitative and quantitative analysis of PCP in aqueous samples.

Acknowledgements This work was supported by the Chinese Academy of Sciences and carried out in the State Key Laboratory of Environmental Aquatic Chemistry.

\section{References}

1. Crosby, D. G., Environmental chemistry of pentachlorophenol, Pure Appl. Chem., 1981, 53: 1051—1080.

2. Zheng, M. H., Zhang, B., Bao, Z. G. et al., Analysis of pentachlorophenol from water, sediments, and fish bile of Dongting Lake in China, Bull. Environ. Contain. Bull. Environ. Contain. Toxicol., 2000, 64: 16-19.

3. Li Hou, Wen, X. J., Tu, C. H. et al., Combination of liquid-phase microextraction and on-column stacking for trace analysis of amino alcohols by capillary electrophoresis, Journal of Chromatography A, 2002, 979: 163-169.

4. Jeannot, M. A., Cantwell, F. F., Solvent microextraction into a single drop, Anal. Chem., 1996, 68: 2236-2240.

5. Jeannot, M. A., Cantwell, F. F., Mass transfer characteristics of solvent extraction into a single drop at the tip of a syringe needle, Anal. Chem., 1997, 69: 235-239.

6. He, Y., Lee, H. K., Liquid-phase microextraction in a single drop of organic solvent by using a conventional microsyringe, 
Anal. Chem., 1997, 69: 4634-4640.

7. Zhao, L. M., Lee, H. K., Application of static liquid-phase microextraction to the analysis of organochlorine pesticides in water, Journal of Chromatography A, 2001, 919: 381-388.

8. Liu Wuping, Lee, H. K., Continuous-flow microextraction exceeding 1000-fold concentration of dilute analytes, Anal. Chem., 2000, 72: 4462-4467.

9. Wang, Y., Kwok, Y. C., He, Y. et al., Application of dynamic liquid-phase microextraction to the analysis of chlorobenzenes in water by using a conventional microsyringe, Anal. Chem., 1998, 70: 4610 - 4614.

10. Solveig, A., Halvorsen, T. G., Stig, P. B., Liquid-phase microextraction combined with capillary electrophoresis, a promising tool for the determination of chiral drugs in biological matrices, Journal of Chromatography A, 2002, 963: 303-312.

11. Tung, S. H., Stig, P B., Knut, E. R., Recovery, enrichment and selectivity in liquid-phase microextraction comparison with conventional liquid-liquid extraction, Journal of Chromatography A, 2002, 963: 3-17.

12. Li, H., Gang, S., Lee, H. K., Automated hollow fiber-protected dynamic liquid-phase microextraction of pesticides for gas chromatography-mass spectrometric analysis, Journal of Chromatography A, 2003, 985: 107-116.

13. Lee, M. R., Yeh, Y. C., Hsiang, W. S. et al., Application of solid-phase microextraction and gas chromatography-mass spectrometry for the determination of chlorophenols in urine, Journal of Chromatography B, 1998, 707: 91—97. 